\section{Genetic Analysis and Related Gene Primary Mapping of Heat Stress Tolerance in Cucumber Using Bulked Segregant Analysis}

\author{
Min Wang, Wenrui Liu, Biao Jiang, and Qingwu Peng \\ Vegetable Research Institute, Guangdong Academy of Agricultural Sciences, \\ Guangzhou 510640, China
}

\author{
Xiaoming He \\ Vegetable Research Institute, Guangdong Academy of Agricultural Sciences, \\ Guangzhou 510640, China; and Guangdong Key Laboratory for New \\ Technology Research of Vegetables, Guangzhou 510640, China
}

\section{Zhaojun Liang and Yu'e Lin ${ }^{1}$ \\ Vegetable Research Institute, Guangdong Academy of Agricultural Sciences, Guangzhou 510640, China}

Additional index words. heat stress, BSA, gene mapping, cucumber

\begin{abstract}
Heat stress (HS) negatively influences plant development and growth, especially production and quality. Cucumber is a widely cultivated plant in the gourd family Cucurbitaceae that is often exposed to high temperatures during summer and protected cultivation. In this study, we performed whole-genome re-sequencing of two pools, one heat-tolerant and one heat-sensitive, of the $F_{2}$ population derived from L-9 (heat-resistant) and A-16 (heat-sensitive). The genetic analysis showed that the heat resistance of $L-9$ cucumber seedlings was controlled by a single recessive gene. By combining bulked segregant analysis (BSA) technology, the crucial gene related to HS was preliminarily mapped to a 1.08-Mb region on chromosome 1. To fine-map the locus, Indel markers were designed according to the genomic sequence. Finally, the gene was narrowed to a 550-kb region flanked by two Indel markers, namely Indel-H90 and Indel-H224, that contained 56 candidate genes. Re-sequencing results indicated that 10 candidate genes among the 56 in the candidate region showed single base pair differences in the exons. Quantitative reversetranscription polymerase chain reaction showed that 6 genes among the 10 candidate genes were significantly decreased when exposed to high temperatures. These results not only were useful for the isolation and characterization of the key genes involved in HS but also provided a basis for understanding the mechanism of heat tolerance regulation.
\end{abstract}

In the recent years, high temperatures due to global warming have led to catastrophic crop losses and widespread famine world-

Received for publication 7 Nov. 2018. Accepted for publication 14 Dec. 2018.

This work was supported by the National Key Research and Development Programme (2018YFD0100700), the Natural Science Foundation of Guangdong Province (2018A030310196), Guangdong Special Project Youth Top-Notch Talent Project (2016TQ03N529), the Presidential Foundation of Guangdong Academy of Agricultural Sciences: (201813), and Key Laboratory Open Fund Project of Vegetable Institution of GAAS (201701).

Min Wang and Yu'e Lin designed the experiment. Min Wang performed most of the experiments. Wenrui Liu, Biao Jiang, Qingwu Peng, Xiaoming $\mathrm{He}$, and Zhaojun Liang participated in revising the paper and performed some experiments. Min Wang wrote the paper. Yu'e Lin edited the paper.

All authors declare that the research was conducted in the absence of any commercial or financial relationships that could be construed as potential conflicts of interest.

${ }^{1}$ Corresponding author. E-mail: cucumber200@163. com. wide (Bita and Gerats, 2013). HS could cause changes in plant respiration and photosynthesis, resulting in shortened life cycles and diminished plant productivity (Hall et al., 2001; Ray et al., 2015). HS causes considerable morphological damage, including scorching and sunburn of leaves, leaf senescence, shoot and root growth inhibition, fruit discoloration, and fruit damage (Ismail and Hall, 1999; Vollenweider and GunthardtGoerg, 2005). It also causes cellular damage species (ROS) and antioxidants, content changes of secondary metabolites, and production of related HS proteins (Wahid et al., 2007; Xu et al., 2006). To survive HS, plants have to regulate the transcription of stressrelated genes, signal transduction pathways (Wahid et al., 2007) such as heat shock proteins (HSPs) (Bowen et al., 2002; Xu et al., 2006) and heat shock transcription factors (HSFs) (Panchuk et al., 2002), and the HS signal transduction pathway (Joyce et al., 2003; Suzuki and Mittler, 2006).

Cucumber (Cucumis sativus L., $2 n=2 x=$ 14 ) is a widely cultivated plant in the gourd such as the accumulation of reactive oxygen family Cucurbitaceae. It originated from the southern Himalayas and prefers to grow in warm conditions (Plader et al., 1998). High temperatures lead to negative impacts on cucumber development, especially production and quality, during summer and protected cultivation (Wahid et al., 2007). Therefore, breeding cucumber cultivars with thermostability is a useful strategy for improving the heat tolerance of plants (Wahid et al., 2007). Furthermore, the identification and characterization of genes involved in HS responses could greatly facilitate the development of improved cucumber cultivars with enhanced heat tolerance.

Multiple studies of the stress response of vegetables under various abiotic stress conditions have been performed (Gous et al., 2016; Wang et al., 2018; Zhou et al., 2013). Studies of HS on vegetables have mainly focused on HSPs and relative enzymes. For instance, the accumulation of small HSPs in peanuts could improve the resistance to HS (Chakraborty et al., 2018). CaHSP24 in pepper is significantly induced by high temperatures, thus leading to its thermotolerance (Zhu et al., 2011). In cucumber, the HSF family was identified and analyzed by a genome-wide assay (Zhou et al., 2016). Under high-temperature stress, ABA could induce the expression of $H S P 70$, leading to its tolerance to heat in cucumber ( $\mathrm{Li}$ et al., 2015). MicroRNAs were related to improvements in the high-temperature tolerance of cucumber seedlings (Wang et al., 2018). Ten quantitative trait loci (QTLs) (rate of contribution, 6\% to $17 \%$ ) influencing HS in cucumber have been detected (Chen et al., 2008). Yang (2006) obtained three QTLs that impact heat tolerance in cucumber with $6.18 \%$ to $10.56 \%$ genetic variability. However, no related gene has been fine-mapped or isolated in cucumber, and little is known about the molecular mechanism involved in HS.

In the present study, we combined the BSA (Michelmore et al., 1991) method and whole-genome re-sequencing to analyze heat-tolerant and heat-sensitive pools in cucumber. The genome-wide SNP analysis allowed us to detect a genomic region that harbored the target gene involved in HS. Genetic analysis and fine-mapping of the HS gene were performed to provide a basis for molecular cloning, the mechanism of heat tolerance, and future marker-assisted selection breeding to develop new heat-resistant cucumber cultivars.

\section{Materials and Methods}

Plant materials. Two cucumber cultivars, L-9 (South China cucumber variety, heatresistant) and A-16 (North China cucumber variety, heat-sensitive), were used as parents for the $F_{2}$ population in this study. Onehundred sixty seeds of parents $F_{1}$ and $F_{2}$ were germinated overnight on a wet filter in a culture dish at $28{ }^{\circ} \mathrm{C}$ in a dark environment. Then, seedlings were grown in a feeding block for $14 \mathrm{~h} / 10 \mathrm{~h}$ at $28{ }^{\circ} \mathrm{C} / 18{ }^{\circ} \mathrm{C}$ during 
day/night, respectively, in a culture room $(5,500$ lux $)$. When plants were grown to the one true leaf stage, they were transferred to HS conditions $\left(14 \mathrm{~h} / 10 \mathrm{~h}\right.$ at $42{ }^{\circ} \mathrm{C} / 36{ }^{\circ} \mathrm{C}$ during day/night) for $7 \mathrm{~d}$. Then, seedlings were recovered for $3 \mathrm{~d}$ under normal conditions. Before HS treatment, normal leaves of parents, $F_{1}$ and $160 F_{2}$ were pooled and sampled, respectively. Then, these samples were immediately frozen in liquid nitrogen and consistently stored at $-80{ }^{\circ} \mathrm{C}$ for further analyses.

Generation of BSA data. Genome DNA of parents $F_{1}$ and $F_{2}$ were isolated using the CTAB method (Murray and Thompson, 1980) and used for the BSA. One heat-tolerant DNA pool ( $\mathrm{T}$ pool) and one heat-sensitive DNA pool (S pool) were constructed by mixing an equal amount of DNA from 30 heat-tolerant and 30 heat-sensitive $F_{2}$ plants. Then, these raw reads were carefully filtered to produce clean reads according to three stringent filtering standards: 1) removing reads with $\geq 10 \%$ unidentified nucleotides $(\mathrm{N}) ; 2$ ) removing reads with $>50 \%$ bases with Phred quality scores $\leq 20$; and 3 ) removing reads aligned to the barcode adapter.

Variants identification and annotation. To identify SNPs and Indels that differed between parents and pools, BurrowsWheeler Aligner (BWA) was used to align the clean reads from each sample against the public reference genome (Li and Durbin, 2009). Variant calling was performed for all samples using the GATK Unified Genotyper. SNPs and Indels were filtered using GATK Variant Filtration with proper standards (Window 4, filter QD $<$ 4.0||FS $>60.0 \| \mathrm{MQ}<40.0$, G_filter GQ $<$ 20); those exhibiting segregation distortion or sequencing errors were discarded. To determine the physical positions of each SNP, the software tool ANNOVAR (Wang et al., 2010) was used to align and annotate SNPs or Indels.

Analysis of BSA data. The SNP index and $\Delta$ (SNP index) were calculated to identify candidate regions for the HS gene (Lu et al., 2014; Takagi et al., 2013). An SNP index is a proportion of reads harboring the SNP that differs from the reference sequence and $\Delta$ (SNP index) when obtained by subtraction of the SNP index of the T pool from that of the S pool. The SNP index represents frequencies of parental alleles in the population of bulked individuals. The SNP index was calculated at all SNP positions by in-house Perl scrips with the following values: SNP index $>3$ and SNP depth $>6$. A slide window analysis was applied to SNP index plots with a $2-\mathrm{Mb}$ window size and $100-\mathrm{kb}$ increments. The $\Delta$ (SNP index) was calculated based on subtraction of the SNP index from two bulk pools. The $\Delta$ (SNP index) was zero in most of the genomic regions, but a few genomic regions exhibited positive or negative $\Delta$ (SNP index) values. The related locus was identified in these positive or negative peak regions (with a $95 \%$ confidence interval) for 10,000 boot strap replicates.
Fine-mapping of the candidate gene. A total of 302 Indel markers that were welldistributed in the candidate region on chromosome 1 were used for the gene linkage analysis. New Indel markers were developed based on the DNA sequence polymorphisms between L-9 and A-16 according to the resequencing genome results.

Quantitative real-time polymerase chain reaction identification. A quantitative realtime polymerase chain reaction (PCR) analysis was performed using the total RNA from seedling leaves of parents and the normal and HS treatments. Additionally, $20 \mu \mathrm{L}$ cDNA was obtained using the QuantiTect Reverse Transcription Kit (Qiagen, Hilden, Germany). Quantitative reverse-transcriptase PCR (qRT-PCR) $(20 \mu \mathrm{L}$ reaction volume) was performed with $0.5 \mu \mathrm{L}$ of cDNA, $0.2 \mu \mathrm{M}$ of primer mix, SYBR Premix Ex Taq Kit (TaKaRa, Tokyo, Japan), and the ABI PRISM 7900HT system (Applied Biosystems, Foster City, CA). Cucumber $\alpha$-TUBULIN (TUA) gene was used normally. All qRT-PCR primers are listed in Table 3.

Statistical analysis. Significant differences were detected by IBM SPSS Statistics 20 (using Student's $t$ test). Relative gene expressions were calculated using the $2^{-\triangle \Delta \mathrm{Ct}}$ method (Jarošová and Kundu, 2010). In addition, GraphPad Prism 5 was used for chart preparation.

\section{Results}

Phenotypes analysis of parents and $F_{2}$ plants. Ten-day seedlings of parents $\mathrm{F}_{1}$ and $\mathrm{F}_{2}$ plants grown under normal conditions (Fig. 1A) were treated with HS for $7 \mathrm{~d}$ and recovered for $3 \mathrm{~d}$ (Fig. 1B). L-9, A-16, and $\mathrm{F}_{1}$ showed vigorous development before treatment; however, A-16 and $F_{1}$ exerted dry wilting after HS, and their leaves turned chlorotic and yellow (Fig. 1A and B). Most $\mathrm{F}_{2}$ plants were sensitive to heat, and leaves died after wilting (Fig. 1C and D).
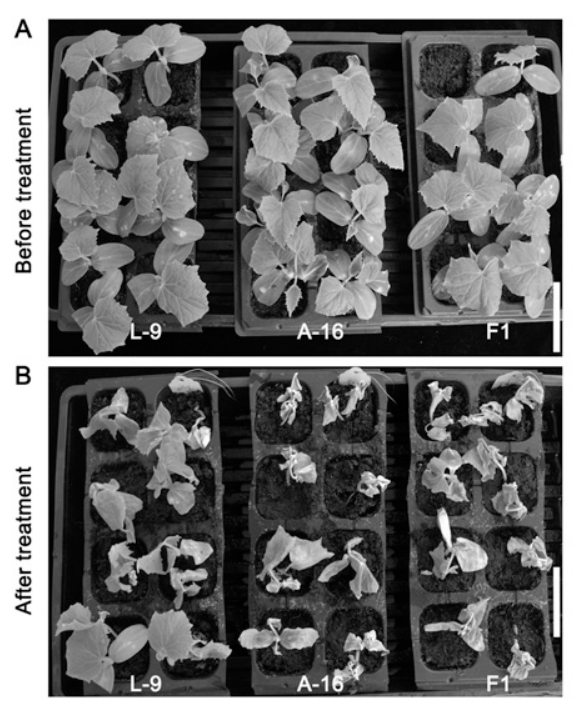

Fig. 1. Phenotype analysis of before and after heat stress (HS). (A and B) L-9, A-16, and $F_{1}$ plants under normal condition (A) and after recovery of HS (B). (C and D) $F_{2}$ phenotype after HS treatment. Bar in (A-D): cm.

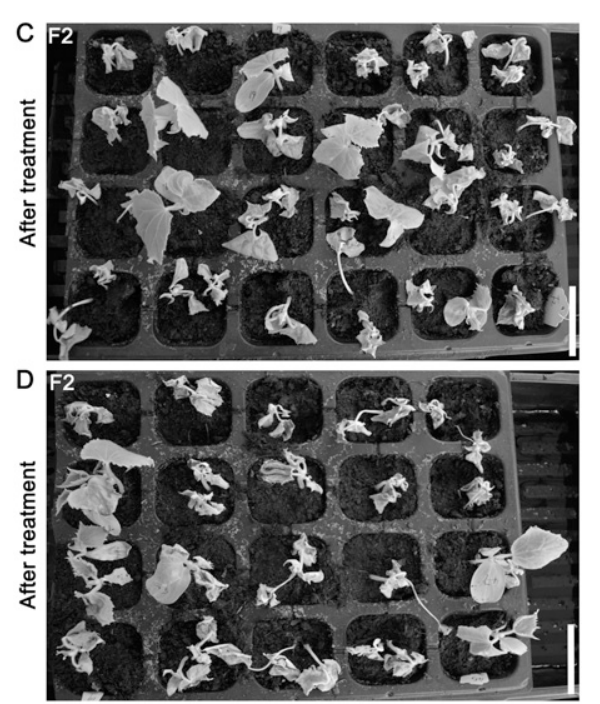

To understand the genetic analysis of cucumber heat tolerance, we analyzed the number of heat-tolerant and heat-sensitive plants. Results showed that all $\mathrm{F}_{1}$ plants were sensitive to heat. Among the $\mathrm{F}_{2}$ population, 112 plants showed sensitivity to high temperatures and 48 plants demonstrated resistance to HS (Table 1), indicating that cucumber thermostability was controlled by a single dominant gene.

Identification of the candidate region by genome re-sequencing. Using Illumina highthroughput sequencing, we finally obtained $65,406,980$ and $61,728,581$ short reads $(100 \mathrm{bp}$ in length) from the $\mathrm{T}$ pool and $\mathrm{S}$ pool, respectively. These short reads were aligned to the 9930 reference genome (Huang et al., 2009), and the average paired mapped reads were $27,516,956$, with an average align ratio of $88.3 \%$ (Table 2). Next, the SNP index was calculated and computed to identify the SNP. SNP index graphs of the T pool (Fig. 2A) and S pool (Fig. 2B) were generated by plotting the average SNP index against the position of each sliding window in the reference genome assembly. The $\delta$ (SNP index) was calculated and plotted against the genome positions (Fig. 2C) by combining the information of the SNP index of these two extreme pools.

Fine-mapping of the candidate gene locus. A previous study reported that SNPs with a SNP index $<0.3$ for two bulked sequences were filtered out during SNP calling to avoid sequencing or alignment errors (Takagi et al., 2013). These band regions of the SNP index for the T pool and $\mathrm{S}$ pool ranged between 0.3 and 0.7 . The region on chromosome 1 from 19.51 to $20.59 \mathrm{Mb}$ (Fig. 3A) was the most probable interval. To fine-map the candidate gene locus, we used the $F_{2}$ genetic population and designed multiple Indel markers in this region. Subsequently, using this mapping strategy, the candidate gene was primarily 
Table 1. Segregation ratios of heat-resistant and heat-sensitive plants in the L-9 $\times$ A-16 genetic population.

\begin{tabular}{lccccc}
\hline Population & Total plants & Sensitivity & Resistance & Expected ratio & $\chi^{2}$ \\
\hline L-9 & 16 & 0 & 16 & - & - \\
A-16 & 16 & 16 & 0 & - & - \\
$F_{1}$ & 16 & 16 & 0 & - & - \\
$F_{2}$ & 160 & 112 & 48 & $3: 1$ & 2.13 \\
\hline
\end{tabular}

Table 2. Comparative statistical analysis between clean reads and paired mapped reads.

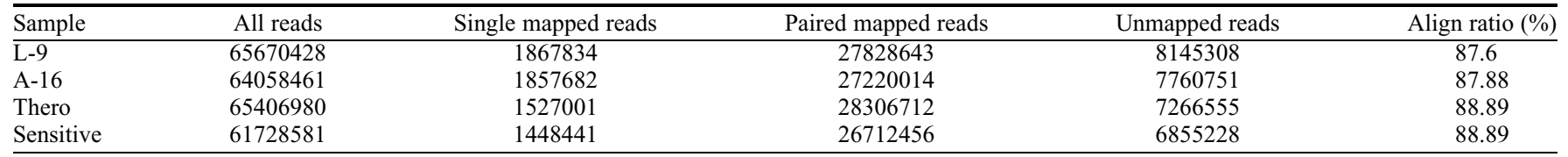

Table 3. Primers used in the gene mapping of the study.

\begin{tabular}{lll}
\hline Primers & \multicolumn{1}{c}{ Forward } & \multicolumn{1}{c}{ Reverse } \\
\hline Indel-H24 & CTTCATTGGCCTAACCCTTG & TGGTTCTTCACGAGGAGTG \\
Indel-H2 & TCAAATTTCATATGCAACATAAATACA & GGATGAAGGAGACATTATTTGC \\
Indel-H37 & TGGTTGTTTGTCTTTACTTTTTGC & TTTTTGAAACGGAGACAAACTTC \\
Indel-H90 & GGTGAGGTGAGGTAAATTGTGTC & TGAAAATTGGGGTTTTTCA \\
Indel-H224 & CAAGATTCAAGAGAGAACTTTTTCG & TTTGCATAAAGAATTTAGGAAGATTA \\
\hline
\end{tabular}
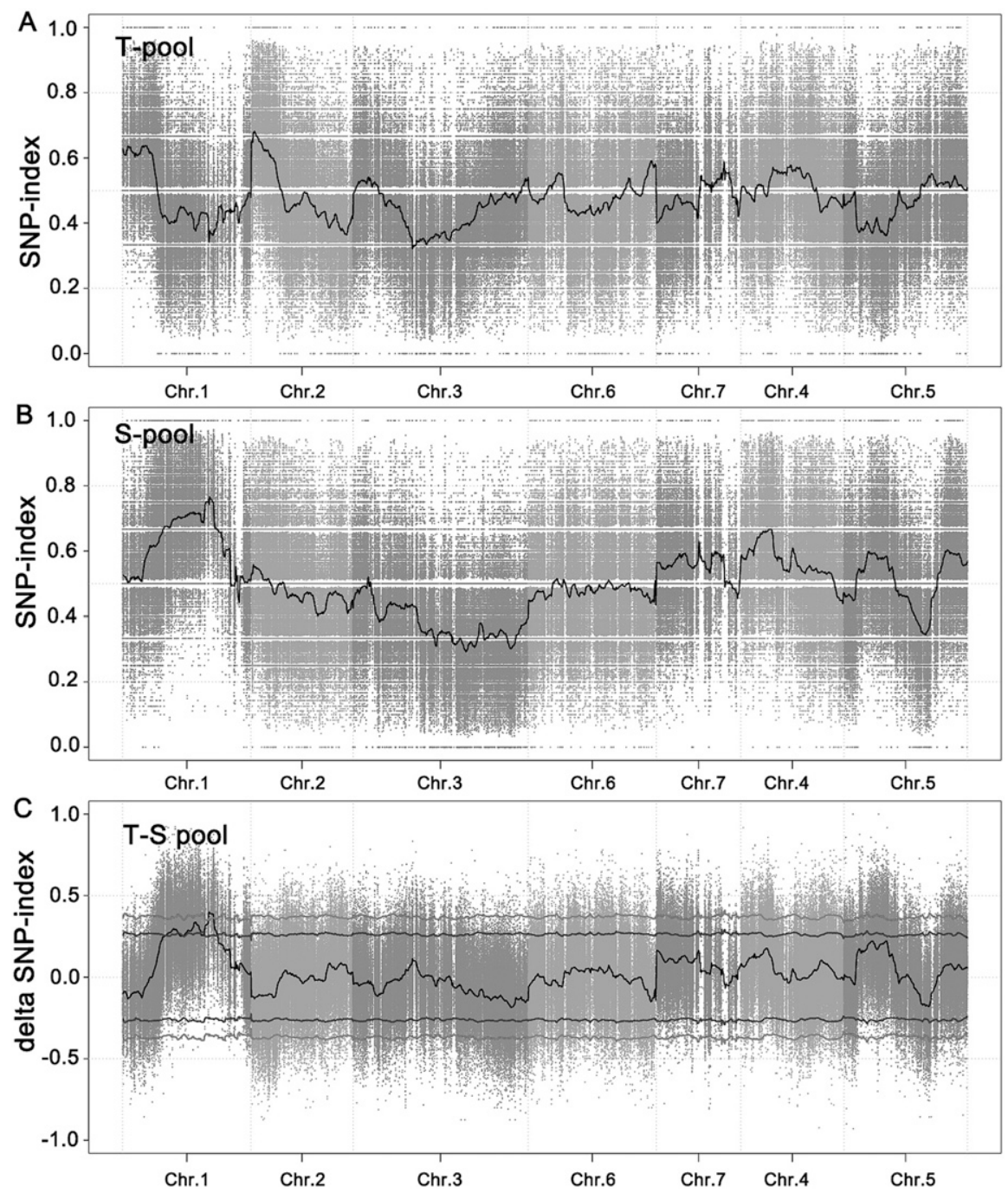

Fig. 2. SNP-index graphs of T-pool (A), S-pool (B), and delta (SNP-index) (C) from sequencing analysis. $\mathrm{X}$-axis represents the position of seven cucumber chromosomes and $\mathrm{Y}$-axis represents the SNP-index.

mapped between two Indel markers, Indel H2 and Indel H224 (Fig. 3B). Next, we used the polymorphism primers to narrow the primary region. Finally, the gene narrowed the locus to a $550-\mathrm{kb}$ region flanked by two Indel markers, Indel H90 and Indel H224 (Fig. 3B; Table 2). The final mapping region contained 56 candidate genes (Fig. 5C). To further narrow the candidate region, we designed many more SSR or Indel markers; however, we were not successful because no polymorphism of these markers was detected in the region.

Analysis of candidate genes related to cucumber HS. After analyzing the bulked segregant sequencing results, we finally mapped the candidate gene in the region of $550 \mathrm{~kb}$ on chromosome 1 . According to the re-sequencing genome data, we found that 10 of these candidate genes showed single differences in the CDS region. Among these 10 candidate genes, only the function of CsalM560770 was unknown. The others functioned in DNA primase/helicase (Csa1M542430), receptor-like protein kinase 4 (CsalM542460), short-chain dehydrogenase/reductase family protein (Csa1M542480), calmodulin-binding protein (Csa1M561930), and other biological pathways (Table 4).

Next, to determine the expression changes under normal and HS conditions, we performed a qRT-PCR assay for these candidate genes. Results showed that 7 of the 10 gene expressions were significantly decreased in A-16 compared with L-9 under HS, whereas two genes showed no difference (Fig. 4). Among them, four gene expressions in L-9 were much higher than that in A-16: CsalM542460 (19.12-times higher), CsalM561420 (24.93-times higher), CsalM561930 (16.78-times higher), and CsalM561940 (15.13-times higher) (Fig. 3). According to previous reports (Kotak et al., 2007; Li et al., 2004; Liu et al., 2005), the gene Csa1M561930, which encodes a calmodulinbinding protein, was the locus most likely related to HS.

Characterization of Csa-HR1.1. Previous studies addressed the possible roles of $\mathrm{Ca}^{2+}$ -dependent signaling during the heat response (review by Kotak et al., 2007) because heat could induce cytosolic $\mathrm{Ca}^{2+}$ transients in Arabidopsis cell cultures with the help of calmodulin (Liu et al., 2005). According to the gene function in the candidate region, only Csa1M561930 encoding a calmodulinbinding protein was related to plant heat resistance; it showed significantly decreased expression in A-16 under HS, possibly as the candidate gene. Therefore, we performed a re-sequenced analysis using the next assay. Results showed that there was a real single base difference between L-9 and A-16, resulting in the change in amino acids 


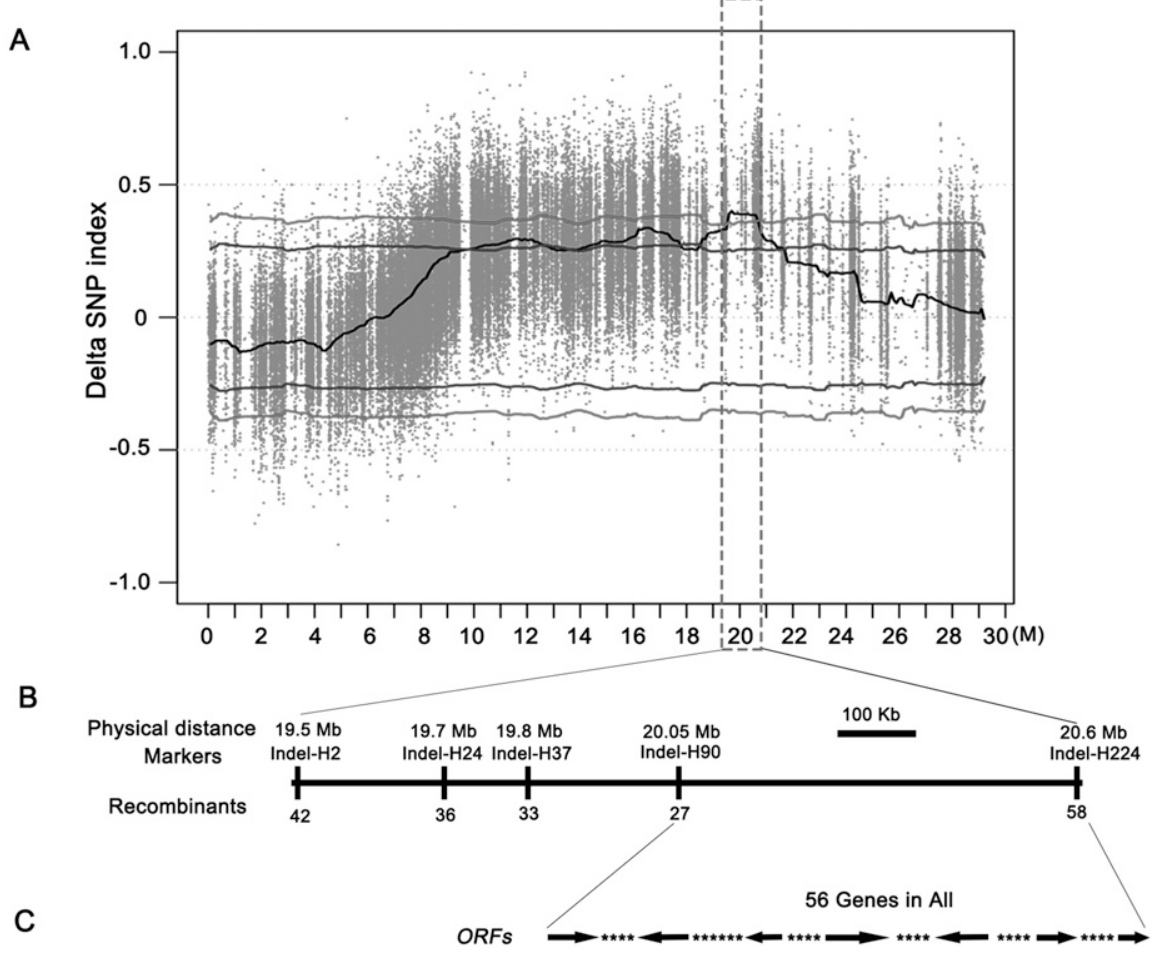

Fig. 3. Delta SNP-index graphs from sequencing analysis. X-axis represents the position of chromosome 1 of cucumber and Y-axis represents the SNP-index. Red box represents the candidate region related to heat stress in cucumber.

Table 4. Analysis of candidate genes related to cucumber heat stress.

\begin{tabular}{lll}
\hline Chromosome & Function gene & \multicolumn{1}{c}{ Description } \\
\hline Chr.1 & CsalM542430 & DNA primase/helicase \\
Chr.1 & CsalM542460 & Putative receptor-like protein kinase 4 \\
Chr.1 & CsalM542480 & Short-chain dehydrogenase/reductase \\
Chr.1 & CsalM554570 & Gamma-soluble NSF attachment protein \\
Chr.1 & Csa1M554580 & Lysine-specific demethylase 5A \\
Chr.1 & CsalM560770 & Unknown protein \\
Chr.1 & Csa1M561360 & Pectin acetylesterase, putative \\
Chr.1 & CsalM561420 & Transcription regulatory protein SNF5 \\
Chr.1 & CsalM561930 & Calmodulin-binding protein \\
Chr.1 & CsalM561940 & Glycine-rich protein \\
\hline
\end{tabular}

(Supplemental Fig. 1A and B). The protein structure included a DUF1645 domain (Supplemental Fig. 1C). To detect the expression of the tissue pattern, we performed qRTPCR. We found that Csa1M561930 was highly expressed in the fruit and flower bud, with lower expression in the stem tendril and cotyledon (Supplemental Fig. 1D).

\section{Discussion}

HS was considered a severely negative factor that led to decreased yield and quality in cereal crops (Bita and Gerats, 2013; Ray et al., 2015). At present, little is known about the molecular mechanism of heat resistance in cucumber, especially because no related genes have been isolated and characterized. In this study, heat tolerance of the cucumber seedling was controlled by a single recessive gene based on the genetic analysis. By combining BSA sequencing technology, we finally mapped the gene related to heat should be performed in future studies to verify our results.

BSA is considered a simple and efficient alternative method for exploring molecular markers linked to target genes or QTLs related to important traits because it genotypes a pair of bulked DNA samples from two sets of individuals with distinct or opposite extreme phenotypes (Giovannoni et al., 1991; Lu et al., 2014; Michelmore et al., 1991; Takagi et al., 2013). For example, QTLs were detected by whole-genome resequencing of DNA from two populations showing extremely opposite traits for a given phenotype in a segregating progeny (Takagi et al., 2013). Candidate genes in cucumber associated with resistance to powdery mildew were identified by combining BSA and SLAF-Seq (Zhang et al., 2015). Furthermore, a major QTL controlling early flowering in cucumber was discovered using next-generation sequencing (Lu et al., 2014). Therefore, in our study, we constructed two pools with opposite heat resistance (heat-tolerant pool and heatsensitive pool) to perform whole-genome resequencing, which offered an efficient way to explore the possible genes related to heat tolerance in cucumber.

To deeply explore the most likely candidate gene related to HS, several molecular SSR or Indel markers were designed in the primary region. Results showed that only a few Indel markers had a polymorphism between L-9 and A-16. Finally, because no Indel polymorphism primers were detected in the $550-\mathrm{kb}$ region, the candidate region could not be further narrowed. After combining sequencing data, we observed 10 out of the 56 candidate genes. Among these 10 genes, Csa1M561930, which encoded a calmodulinbinding protein, was the locus possibly related to HS. Previous studies have demonstrated that CaMK plays a crucial role in plant heat tolerance (Kotak et al., 2007; Liu et al., 2005). $\mathrm{Ca}^{2+}$ transients were only detected after recovery of HS in Arabidopsis (Larkindale et al., 2005). In addition, $\mathrm{Ca}^{2+}$ could increase in vitro DNA binding with HSFs (Li et al., 2004). Here, CsalM561930 was detected based on the difference between L-9 and A-16, which indicated that the gene might be the locus for cucumber HS. Although CsalM561930 in this study was considered the possible gene for cucumber HS, we could not rule out the possibility of other candidate genes that also showed single base pair differences between parents. For example, CsalM542460, which encodes putative receptor-like protein kinase 4 , was reported to be involved in abiotic and biotic stresses such as bacterial pathogen Pseudomonas syringae (Chen et al., 2003), cold, and salt (Hong, 1997). Csa1M542480, which encodes a shortchain dehydrogenase/reductase, was reported to be related to plant defense responses (Hwang et al., 2012). However, these candidate genes, except for CsalM561930, were not directly involved in HS and had certain roles in other abiotic environmental stresses.

We first performed BSA sequencing to fine-map the crucial gene related to HS. After 

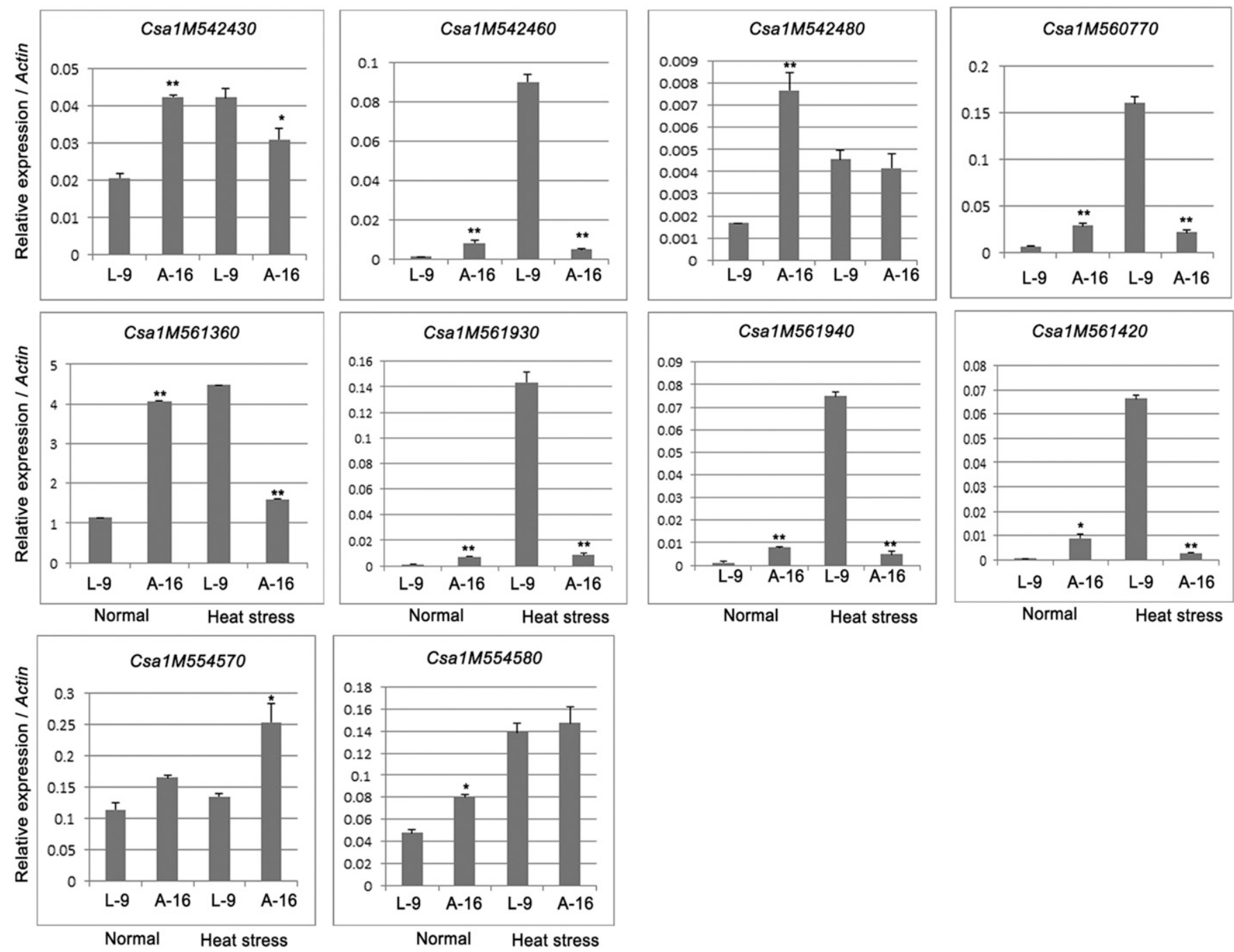

Fig. 4. Expression of candidate genes under normal and heat stress conditions. Data is presented as the mean \pm standard deviation $(\mathrm{n}=9$ ). $* 0.01 \leq P \leq 0.05$, $* * P \leq 0.01$, Student's $t$ test.

combining the genetic analysis and wholegenome re-sequencing, the key gene was located in the $550-\mathrm{kb}$ region flanked with Indel H90 and Indel H224 on chromosome 1. Our study provides a foundation for the further isolation and characterization of key genes involved in HS and provides useful information for understanding molecular regulation of heat tolerance.

\section{Literature Cited}

Bita, C.E. and T. Gerats. 2013. Plant tolerance to high temperature in a changing environment, scientific fundamentals and production of HStolerant crops. Front. Plant Sci. 4:1.

Bowen, J., L.Y. Michael, K.I.M. Plummer, and I.A.N. Ferguson. 2002. The heatshock response is involved in thermotolerance in suspensioncultured apple fruit cells. J. Plant Physiol. 159:599-606.

Chakraborty, K., S.K. Bishi, A.L. Singh, P.V. Zala, M.K. Mahatma, K.A. Kalariya, and R.A. Jat. 2018. Rapid induction of small heat shock proteins improves physiological adaptation to high temperature stress in peanut. J. Agron. Crop Sci. 204:285-297.

Chen, F.X., G.H. Zhang, W.C. Qian, Y.K. Han, D.F. Chen, S.L. Du, and X.W. Chen. 2008.
Molecular markers linked to high temperature resistance QTLs in cucumber. Acta Sci. Natur. Univ. Nankaiensis 41:49-54. (In Chinese).

Chen, K., L. Du, and Z. Chen. 2003. Sensitization of defense responses and activation of programmed cell death by a pathogen-induced receptor-like protein kinase in arabidopsis. Plant Mol. Biol. 53:61-74.

Giovannoni, J.J., R.A. Wing, M.W. Ganal, and S.D. Tanksley. 1991. Isolation of molecular markers from specific chromosome intervals using DNA pools from existing mapping populations. Nucleic Acids Res. 19:6553-6558.

Gous, P.W., L. Hickey, J.T. Christopher, J. Franckowiak, and G.P. Fox. 2016. Discovery of QTL for stay-green and heat-stress in barley (Hordeum vulgare) grown under simulated abiotic stress conditions. Euphytica 207:305-317.

Hall, A.E. 2001. Crop responses to environment. CRC Press LLC, Boca Raton, FL.

Hong, S. 1997. Identification of a receptor-like protein kinase gene rapidly induced by abscisic acid, dehydration, high salt, and cold treatments in arabidopsis thaliana. Plant Physiol. 113(4):1203-1212.

Huang, S.W., R.Q. Li, Z.H. Zhang, L. Li, X.F. Gu, and W. Fan, et al. 2009. The genome of the cucumber, Cucumis sativus L. Nat. Genet. 41:1275-1281.
Hwang, S.G., N.C. Lin, Y.Y. Hsiao, C.H. Kuo, P.F. Chang, W.L. Deng, M.H. Chiang, H.L. Shen, C.Y. Chen, and W.H. Cheng. 2012. The Arabidopsis short-chain dehydrogenase/reductase 3, an abscisic acid deficient 2 homolog, is involved in plant defense responses but not in aba biosynthesis. Plant Physiol. Biochem. 51:6373.

Ismail, A.M. and A.E. Hall. 1999. Reproductivestage heat tolerance, leaf membrane thermostability and plant morphology in cowpea. Crop Sci. 39:1762-1768.

Jarošová, J. and J.K. Kundu. 2010. Validation of reference genes as internal control for studying viral infections in cereals by quantitative realtime RT-PCR. BMC Plant Biol. 10:146.

Joyce, S.M., A.C. Cassells, and J.S. Mohan. 2003. Stress and aberrant phenotypes in vitro culture. Plant Cell Tissue Organ Cult. 74:103121.

Kotak, S., J. Larkindale, U. Lee, P. von KoskullDöring, E. Vierling, and K.D. Scharf. 2007. Complexity of the HS response in plants. Curr. Opin. Plant Biol. 10:310-316.

Larkindale, J., M. Mishkind, and E. Vierling. 2005. Plant responses to high temperature, p. 100 144. In: Plant abiotic stress. Blackwell Publishing.

Li, B., H.T. Liu, D.Y. Sun, and R.G. Zhou. 2004. $\mathrm{Ca}^{2+}$ and calmodulin modulate DNA-binding 
activity of maize heat shock transcription factor in vitro. Plant Cell Physiol. 45:627-634.

Li, H., S.S. Liu, C.Y. Yi, F. Wang, J. Zhou, X.J. Xia, K. Shi, Y.H. Zhou, and J.Q. Yu. 2015. Hydrogen peroxide mediates abscisic acidinduced HSP70 accumulation and heat tolerance in grafted cucumber plants. Plant Cell Environ. 37:2768-2780.

Li, H. and R. Durbin. 2009. Fast and accurate short read alignment with Burrows-Wheeler transform. Bioinformatics 14:1754-1760.

Liu, H.T., D.Y. Sun, and R.G. Zhou. 2005. $\mathrm{Ca}^{2+}$ and AtCaM3 are involved in the expression of heat shock protein gene in Arabidopsis. Plant Cell Environ. 28:1276-1284.

Lu, H., T. Lin, J. Klein, S. Wang, J. Qi, Q. Zhou, J. Sun, Z. Zhang, Y. Weng, and S. Huang. 2014. QTL-seq identifies an early flowering QTL located near flowering locus $\mathrm{T}$ in cucumber. Theor. Appl. Genet 127:1491-1499.

Michelmore, R.W., I. Paran, and R.V. Kesseli. 1991. Identification of markers linked to disease resistance genes by bulked segregant analysis, a rapid method to detect markers in specific genomic regions by using segregating populations. Proc. Natl. Acad. Sci. USA 88:9828-9832.

Murray, M. and W.F. Thompson. 1980. Rapid isolation of high molecular weight plant DNA. Nucleic Acids Res. 8:4321-4326.

Panchuk, I.I., R.A. Volkov, and F. Schoffl. 2002. HS- and heat shock transcription factor- dependent expression and activity of ascorbate peroxidase in Arabidopsis. Plant Physiol. 129: $838-853$.
Plader, W., S. Malepszy, W. Burza, and Z. Rusinowski. 1998. The relationship between the regeneration system and genetic variability in the cucumber (Cucumis sativus L.). Euphytica 103:9-15.

Ray, D.K., J.S. Gerber, G.K. MacDonald, and P.C. West. 2015. Climate variation explains a third of global crop yield variability. Nat. Commun. 6:5989.

Takagi, H., A. Abe, K. Yoshida, S. Kosugi, S. Natsume, C. Mitsuoka, A. Uemura, H. Utsushi, M. Tamiru, S. Takuno, H. Innan, L.M. Cano, S. Kamoun, and R. Terauchi. 2013. QTL-seq, rapid mapping of quantitative trait loci in rice by whole genome resequencing of DNA from two bulked populations. Plant J. 74:174-183.

Suzuki, N. and R. Mittler. 2006. Reactive oxygen species and temperature stresses, a delicate balance between signaling and destruction Physiol. Plant. 126:45-51.

Vollenweider, P. and M.S. Gunthardt-Goerg. 2005. Diagnosis of abiotic and biotic stress factors using the visible symptoms in foliage. Environ. Pollut. 137:455-465.

Wahid, A., S. Gelani, M. Ashraf, and M.R. Foolad. 2007. Heat tolerance in plants: An overview. Environ. Expt. Bot. 61:199-223.

Wang, K., L. Mingyao, and H. Hakon. 2010. ANNOVAR, functional annotation of genetic variants from high-throughput sequencing data. Nucleic Acids Res. 38:e164.

Wang, Y., S. Guo, L. Wang, X. He, S. Shu, J. Sun, and N. Lu. 2018. Identification of microRNAs associated with the exogenous spermidinemediated improvement of high-temperature tolerance in cucumber seedlings (Cucumis sativus L.). BMC Genomics 19:285.

Xu, S., J. Li, X. Zhang, H. Wei, and L. Cui. 2006. Effects of heat acclimation pretreatment on changes of membrane lipid peroxidation, antioxidant metabolites, and ultrastructure of chloroplasts in two cool-season turfgrass species under HS. Environ. Expt. Bot. 56:274285 .

Yang, D.F. 2006. Mapping quantitative traits for the heat tolerance in cucumber. Master Thesis (In Chinese).

Yu, S.C. and Y.J. Wang. 2003. Inherence of heat tolerance in cucumber. Huabei Nongxuebao 18:87-89. (In Chinese).

Zhang, P., Y. Zhu, L. Wang, L. Chen, and S. Zhou. 2015. Mining candidate genes associated with powdery mildew resistance in cucumber via super-BSA by specific length amplified fragment (SLAF) sequencing. BMC Genomics $16: 1058$.

Zhang, P. and Z.W. Qian. 2007. Genetic analysis of heat tolerance in cucumber. J. Northeast Agr. Univ. 38:486-490. (In Chinese).

Zhou, S., P. Zhang, Z. Jing, and J. Shi. 2013. Genome-wide identification and analysis of heat shock transcription factor family in cucumber (Cucumis sativus L.). Plant Omics Journal of Plant Molecular Biology \& Omics 6:449-455.

Zhu, W., M. Lu, Z. Gong, and R. Chen. 2011. Cloning and expression of a small heat shock protein gene CaHSP24 from pepper under abiotic stress. Afr. J. Biotechnol. 1025:49684976. 


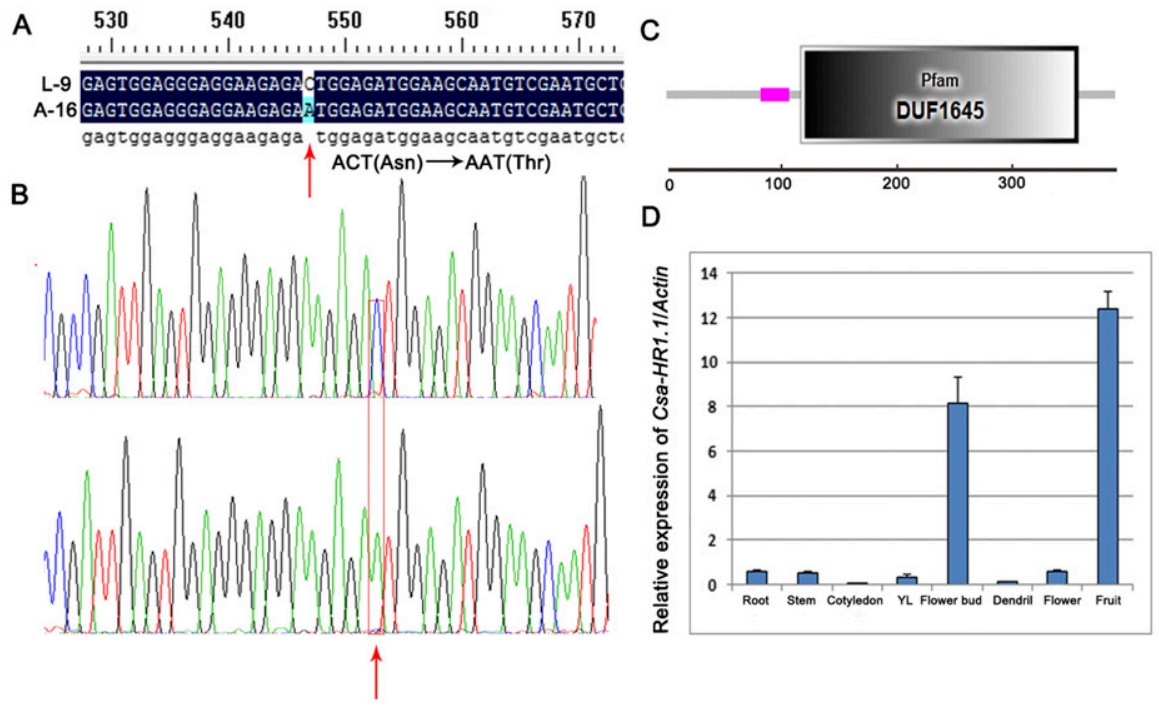

Supplemental Fig. 1. Characterization and tissue expression of Csa1M561930. (A and B) Csa1M561930 showed single base difference between L-9 and A-16. (C) Analysis of Csa1M561930 protein domain. (D) Relative expression of Csa1M561930 in different tissues. Red box represents the mutated base pair. 